\title{
Finite to Infinite - An Expository on Nonstandard Analysis
}

\author{
Alagu. $\mathbf{S}^{*}, \mathbf{R} . \mathrm{Kala}^{2}$ \\ ${ }^{1}$ Department of Mathematics, Manonmaniam Sundaranar University Tirunelveli, Tamilnadu, India \\ ${ }^{2}$ Department of Mathematics, Manonmaniam Sundaranar University Tirunelveli, Tamilnadu, India
}

Corresponding Author: alagu391@gmail.com, phone : 9442331630

Available online at: www.isroset.org

Received: 8/May/2018, Revised: 19/May/2018, Accepted: 5/Jun/2018, Online: 30/Jun/2018

\begin{abstract}
Nonstandard analysis is a branch of Mathematics introduced by Abraham Robinson ${ }^{[I]}$ in 1966. In 1977, Edward Nelson ${ }^{[2]}$ gave an axiomatic approach to Non-standard analysis. In many instances, analysis on infinite sets can be reduced to a finiteness argument using Nonstandard methods. In this expository article, we present an introduction to the theory and indicate an application to infinite graphs. The application is to a result by De Bruijn and Paul R. Erdos that it is enough to study colouring problem only in finite graphs.
\end{abstract}

Keywords- Nonstandard analysis, Graphs, Colouring

\section{INTRODUCTION}

Abraham Robinson constructed a superstructure to work in any given structure like the Euclidean spaces, topological spaces, algebraic structures (rings, fields etc., .), graphs and so on. Instead, Edward Nelson restructured the axiomatics of set theory by introducing three new principles (IST) Idealization, Standardization, Transfer - to the Zermelo Fraenkel set of axioms with the axiom of choice (ZFC) . Nelson proved the consistency of the new system (IST + ZFC). This allows standard and nonstandard elements to work within sets.

First we present the axioms and discuss the immediate outcomes as in [3]. Finally we present an application to Graph Colourings.

\section{AXIOMATICS}

Henceforth whatever we refer to as 'classical' is anything we have come across in Mathematics so far. For instance, sets, cartesian products of sets, relations and functions studied so far, all axioms, mathematical structures and results in classical set theory (ZFC) still hold in our extended analysis - namely Nonstandard analysis. Like the binary predicate ' $\epsilon$ ' (belongs to) and its governing rules in classical set theory, ${ }^{[2]}$ Nelson introduces a unary predicate (for example, complement operation in sets is a unary predicate in classical set theory) 'standard' and spells out its governing rules in the following three axioms. We present the axioms with some discussions in between. The fundamentals are as in Alain Robert ${ }^{[3]}$.
Idealization (I) : Let $\mathrm{R}(\mathrm{x}, \mathrm{y})$ be a classical relation between two sets $\mathrm{X}$ and $\mathrm{Y}$, that is, $\mathrm{R} \subseteq \mathrm{X} \times \mathrm{Y}$. If for every standard and finite $\mathrm{F} \subseteq \mathrm{X}$, there exists $\mathrm{y}_{\mathrm{F}} \in \mathrm{Y}$ such that $\mathrm{R}\left(\mathrm{x}, \mathrm{y}_{\mathrm{F}}\right) \forall \mathrm{X}$ $\in \mathrm{F}$, then there exists $\mathrm{y} \in \mathrm{Y}$ such that $\mathrm{R}(\mathrm{x}, \mathrm{y})$ for all standard $\mathrm{x} \in \mathrm{X}$.

We use the symbols $\forall^{\mathrm{s}}, \exists^{\mathrm{s}}, \forall^{\mathrm{f}}, \exists^{\mathrm{f}}, \forall^{\text {sf }}$ and $\exists^{\text {sf }}$ to mean 'for every standard', 'there exists standard', 'for every finite', 'there exists finite', 'for every standard finite' and 'there exists standard finite' respectively. Hence restated Idealization axiom is as follows.

Let $\mathrm{R}(\mathrm{x}, \mathrm{y})$ be a classical relation between two sets $\mathrm{X}$ and $\mathrm{Y}$. That is, $\mathrm{R} \subseteq \mathrm{X} \times \mathrm{Y} . \forall^{\text {sf }} \mathrm{F} \subseteq \mathrm{X}, \exists \mathrm{y}_{\mathrm{F}} \in \mathrm{Y}$ such that $\mathrm{R}(\mathrm{x}$, $\left.\mathrm{y}_{\mathrm{F}}\right) \forall \mathrm{x} \in \mathrm{F} \Rightarrow \exists \mathrm{y} \in \mathrm{Y}$ such that $\mathrm{R}(\mathrm{x}, \mathrm{y}) \forall^{\mathrm{s}} \mathrm{x} \in \mathrm{X}$.

We discuss some consequences before proceeding to the next two axioms. An element which is not standard will be called nonstandard.

Consequence 1. Every infinite set has nonstandard elements.

Proof. Let $\mathrm{X}$ be an infinite set and $\mathrm{R}$ be a relation on $\mathrm{X}$ defined by $R(x, y)$ if $x \neq y$. Now $\forall{ }^{\text {sf }} F \subseteq X, \quad \exists y_{F} \in X$ such that $R\left(x, y_{F}\right)$, since $X$ is infinite. $B y(I), \exists y \in X$ such that $R(x, y) \forall^{s} x \in X$. That is, $\quad y \neq x \forall^{s} x \in X$. This $y$ must be nonstandard.

Consequence 2. Given any set $X$, there exists a finite subset $\mathrm{F}$ of $\mathrm{X}$ containing all standard elements of $\mathrm{X}$. 
Proof. Let $\mathrm{P}_{\mathrm{f}}(\mathrm{X})$ denote the collection of all finite subsets of $X$. Let $R$ be a relation between $X$ and $P_{f}(X)$ defined by $\mathrm{R}(\mathrm{x}, \mathrm{A})$ if $\mathrm{x} \in \mathrm{A} . \forall^{\mathrm{sf}} \mathrm{A} \subseteq \mathrm{X}, \quad \exists \mathrm{A} \in \mathrm{P}_{\mathrm{f}}(\mathrm{X})$ such that $\mathrm{R}(\mathrm{x}, \mathrm{A}) \forall \mathrm{x} \in \mathrm{A}$. By (I), $\exists \mathrm{F} \in \mathrm{P}_{\mathrm{f}}(\mathrm{X})$ such that $\mathrm{R}(\mathrm{x}, \mathrm{F})$ $\forall^{\mathrm{s}} \mathrm{x} \in \mathrm{X}$. That is, $\mathrm{x} \in \mathrm{F} \forall^{\mathrm{s}} \mathrm{x} \in \mathrm{X}$. Hence proved.

Next we move to Standardization axiom.

Standardization $(\boldsymbol{S})$ : Let $\mathrm{P}$ be a property (classical or not) on a standard set $\mathrm{X}$. Then there exists a unique standard subset $E$ of $X$ such that the standard elements of $E$ are precisely the standard elements of $\mathrm{X}$ satisfying the property P. E is denoted by

${ }^{S}\{x \in X / P(x)\}$.

It will be appropriate to discuss consequences of Standardization axiom after presenting Transfer axiom and some of its consequences.

Transfer $(\boldsymbol{T})$ : Let $\mathrm{F}$ be a formula involving a variable $\mathrm{x}$ and standard parameters A,B,C etc.,. Then

$\left(\forall^{\mathrm{s}} \mathrm{x}\right)[\mathrm{F}(\mathrm{x}, \mathrm{A}, \mathrm{B}, \mathrm{C} \ldots)] \Leftrightarrow(\forall \mathrm{x})[\mathrm{F}(\mathrm{x}, \mathrm{A}, \mathrm{B}, \mathrm{C} \ldots)](*)$. Applying it for the negation $\neg \mathrm{F}$ of $\mathrm{F}$, we get

$\left(\forall^{\mathrm{s}} \mathrm{x}\right)[\neg \mathrm{F}(\mathrm{x}, \mathrm{A}, \mathrm{B}, \mathrm{C} \ldots)] \Leftrightarrow(\forall \mathrm{x})[\neg \mathrm{F}(\mathrm{x}, \mathrm{A}, \mathrm{B}, \mathrm{C} \ldots)]$. This is equivalent to the dual Transfer Principle.

$\left(\exists^{\mathrm{s}} \mathrm{x}\right)[\mathrm{F}(\mathrm{x}, \mathrm{A}, \mathrm{B}, \mathrm{C} \ldots)] \Leftrightarrow(\exists \mathrm{x})[\mathrm{F}(\mathrm{x}, \mathrm{A}, \mathrm{B}, \mathrm{C} \ldots)](* *)$.

Thus the Transfer principle is valid with the existential quantifier ' $\exists$ ' in place of the universal quantifier ' $\forall$ ' too.

Consequence 3. The Transfer may be extended to any finite number of quantifiers. For instance, if $A, B, C$..... are standard parameters, $(\forall \mathrm{x})(\forall \mathrm{y}) \ldots . .[\mathrm{F}(\mathrm{x}, \mathrm{y}, \ldots \mathrm{A}, \mathrm{B} \ldots .).] \Leftrightarrow$ $\left.\left(\forall^{\mathrm{s}} \mathrm{x}\right)\left(\forall^{\mathrm{s}} \mathrm{y}\right) \ldots . . \mathrm{F}(\mathrm{x}, \mathrm{y}, \ldots \mathrm{A}, \mathrm{B} \ldots).\right]$.

Consequence 4. In view of (**), whenever an entity is uniquely established in classical theory, this entity must be standard. Thus the numbers like $3,-101 / 103, e, \pi, \sqrt{2}$, the set of natural numbers $N$, the set of real numbers $R$ etc.,. are all standard.

Consequence 5. Let $A, B$ be standard sets. To show $A \subseteq B$, it is enough to check $x \in A \Rightarrow x \in B$ for standard elements. This follows from Transfer axiom: $\left(\forall^{s} x\right)[x \in A \Rightarrow x \in B]$ $\Leftrightarrow(\forall x)[x \in A \Rightarrow x \in B]$. Thus two standard sets are equal if both have the same standard elements.

Consequence 6. In a set E every element is standard if and only if $E$ is a finite and standard set.

Proof. $(\exists \mathrm{x} \in \mathrm{E})[\mathrm{x}$ is nonstandard]

$\Leftrightarrow(\exists \mathrm{x} \in \mathrm{E})\left(\forall^{\mathrm{s}} \mathrm{y} \in \mathrm{E}\right)[\mathrm{y} \neq \mathrm{x}]$

$\Leftrightarrow\left(\forall^{\mathrm{sf}} \mathrm{F}\right)(\exists \mathrm{x} \in \mathrm{E})(\forall \mathrm{y} \in \mathrm{F})[\mathrm{y} \neq \mathrm{x}]$, by $(\mathrm{I})$.

$\Leftrightarrow\left(\forall^{\text {sf }} \mathrm{F}\right)(\exists \mathrm{x} \in \mathrm{E})[\mathrm{x} \notin \mathrm{F}]$

$\Leftrightarrow\left(\forall^{\mathrm{sf}} \mathrm{F}\right)[\mathrm{E} \nsubseteq \mathrm{F}]$

Negating the above equivalence, $(\forall \mathrm{x} \in \mathrm{E})$ [ $\mathrm{x}$ is standard] $\Leftrightarrow \exists^{\text {sf }} \mathrm{F}[\mathrm{E} \subseteq \mathrm{F}](*)$

We shall use the above equivalence $(*)$ to establish the statement of consequence 6 .

If $\mathrm{E}$ is standard and finite, taking $\mathrm{F}=\mathrm{E}$, the implication $\Longleftarrow$ of $(*)$ gives that every $\mathrm{x} \in \mathrm{E}$ is standard.

Conversely let every $\mathrm{x} \in \mathrm{E}$ be standard. Then $\Rightarrow$ of (*) gives a standard, finite $F$ such that $\mathrm{E} \subseteq \mathrm{F}$. First of all this says $\mathrm{E}$ is finite. $\mathrm{F}$ is standard and finite implies the power set $\mathrm{P}(\mathrm{F})$ is standard and finite. $\mathrm{P}(\mathrm{F})$ is standard (by Consequence 4). By what we have established, every element of $P(F)$ is standard and hence $\mathrm{E}$ is standard.

\section{AN APPLICATION}

As a finishing touch we wish to give an application to infinite graphs. It explains why it is enough to study colouring problem only in finite graphs. A Nonstandard proof of it is by De Bruijn and Paul R.Erdos as presented in [4] and is based on Robinson's ultrapower construction. We present a nonstandard proof based on Nelson's Internal Set theory.

Theorem 2.1. If every finite subgraph of an infinite graph $G$ is $k$-colourable, then $G$ itself is $k$ colourable.

Proof. First we assume $\mathrm{G}$ is a standard graph. Let $\mathrm{F}$ $\subseteq \mathrm{V}(\mathrm{G})$ such that $\mathrm{F}$ is finite and contains all standard elements of $V(G)$. Since $F$ is finite, $\exists \mathrm{f}$ : $\mathrm{F} \rightarrow\{1,2, \ldots \mathrm{k}\}$ such that $\mathrm{x}, \mathrm{y} \in \mathrm{F}$ and $\langle x, y\rangle \in \mathrm{E}$ $(\mathrm{G}) \Rightarrow \mathrm{f}(\mathrm{x}) \neq \mathrm{f}(\mathrm{y})$. Extend $\mathrm{f}$ to $\mathrm{V}(\mathrm{G})$ in any way. We have $(\exists \mathrm{f}: \mathrm{V}(\mathrm{G}) \rightarrow\{1,2, \ldots \mathrm{k}\})\left(\forall^{\mathrm{s}} \mathrm{x}, \mathrm{y} \in \mathrm{V}\right.$ (G) $[\langle x, y\rangle \in \mathrm{E}(\mathrm{G}) \Rightarrow \mathrm{f}(\mathrm{x}) \neq \mathrm{f}(\mathrm{y})]$. By transfer $(\exists$ $\mathrm{s} \quad \mathrm{f}: \mathrm{V}(\mathrm{G}) \rightarrow\{1,2, \ldots \mathrm{k}\})\left(\forall^{\mathrm{s}} \mathrm{x}, \mathrm{y} \in \mathrm{V}(\mathrm{G})\right)$ $[\langle x, y\rangle \in \mathrm{E}(\mathrm{G}) \Rightarrow \mathrm{f}(\mathrm{x}) \neq \mathrm{f}(\mathrm{y})]$, since the parameters involved are all standard.

$\left(\forall^{\mathrm{s}} \operatorname{graph} \mathrm{G}\right)\left(\exists^{\mathrm{s}} \mathrm{f}: \mathrm{V}(\mathrm{G}) \rightarrow\{1,2, \ldots \mathrm{k}\}\right)\left(\forall^{\mathrm{s}} \mathrm{x}, \mathrm{y}\right.$ $\in \mathrm{V}(\mathrm{G}))[\langle x, y\rangle \in \mathrm{E}(\mathrm{G}) \Rightarrow \mathrm{f}(\mathrm{x}) \neq \mathrm{f}(\mathrm{y})]$.

Again by Transfer,

$(\forall$ graph G) $(\exists \mathrm{f} \mathrm{:} \mathrm{V}(\mathrm{G}) \rightarrow\{1,2, \ldots . \mathrm{k}\})(\forall \mathrm{x}, \mathrm{y} \in$

$\mathrm{V}(\mathrm{G}))[\langle x, y\rangle \in \mathrm{E}(\mathrm{G}) \Rightarrow \mathrm{f}(\mathrm{x}) \neq \mathrm{f}(\mathrm{y})]$ This completes the proof.

\section{ACKNOWLEDGMENT}

The first author thanks Department of Science and 
Technology (DST), India, for its financial support through INSPIRE fellowship.

\section{REFERENCES}

[1] Abraham Robinson, Nonstandard Analysis, North Holland Publishing Company, 1966.

[2] Edward Nelson, Internal Set theory, a new approach to NSA, Bull. Amer. Math. Soc.,83(1977) pp.1165-1198.

[3] Alain Robert, Nonstandard Analysis, John Wiley and sons, 1985.

[4] A.E.Hurd and P.A.Loeb, An Introduction to Non Standard Real Analysis, Academic Press (1985).

\section{AUTHORS' PROFILE}

Alagu. S, (M.Sc., M.Phil.) is a Research Scholar pursuing Ph.D degree in Mathematics at Manonmaniam Sundaranr University, Tirunelveli. She gave a brilliant academic performance securing first rank at the university level in both undergraduate and postgraduate studies. Presently, she is a DST INSPIRE fellow.

Dr. R. Kala is a Professor of Mathematics at Manonmaniam Sundaranar University, Tirunelveli. She has published 3 books, 45 papers in International journals and 9 in national journals. She has 19 years of teaching experience and 25 years of research experience.
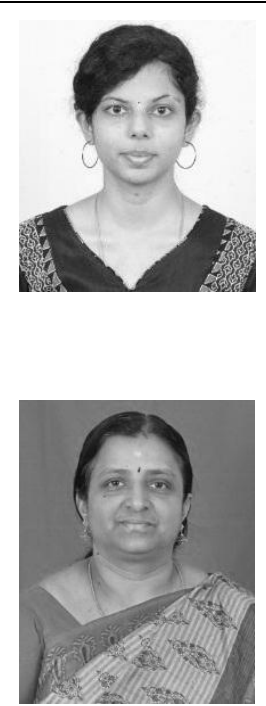